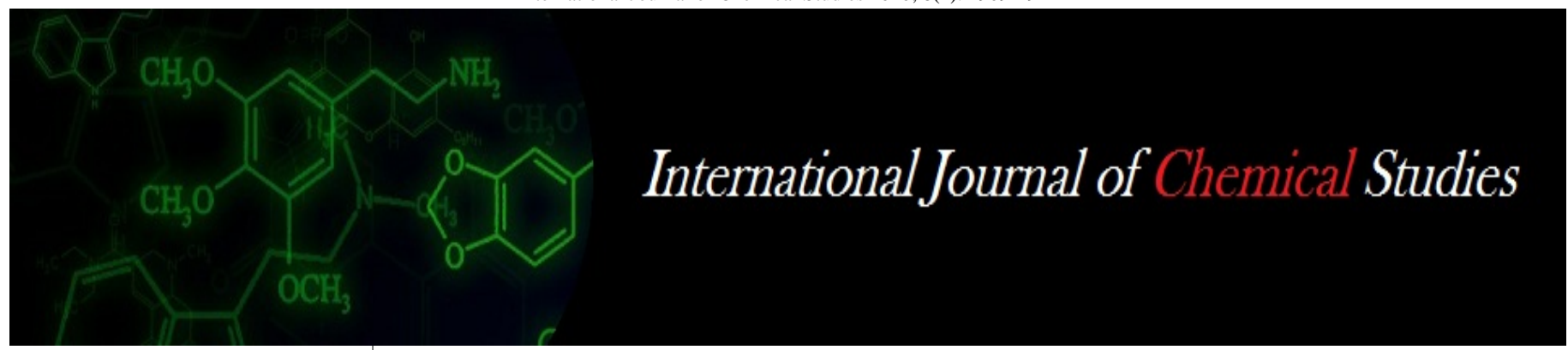

P-ISSN: 2349-8528

E-ISSN: 2321-4902

www.chemijournal.com

IJCS 2020; 8(4): 2909-2912

(C) 2020 IJCS

Received: 18-05-2020

Accepted: 21-06-2020

PC Yadav

Department of Genetics and Plant Breeding, Chandra

Shekhar Azad University of Agriculture and Technology, Kanpur, Uttar Pradesh, India

\section{RK Yadav}

Department of Genetics and Plant Breeding, Chandra Shekhar Azad University of Agriculture and Technology, Kanpur, Uttar Pradesh, India

Vishawanath

Department of Genetics and Plant Breeding, Chandra Shekhar Azad University of Agriculture and Technology, Kanpur, Uttar Pradesh, India

\section{Laxmi Prajapati}

Department of Genetics and Plant Breeding, Chandra Shekhar Azad University of Agriculture and Technology, Kanpur, Uttar Pradesh, India

Corresponding Author: PC Yadav

Department of Genetics and Plant Breeding, Chandra Shekhar Azad University of Agriculture and Technology, Kanpur, Uttar Pradesh, India

\section{Stability analysis over environments in linseed (Linum usitatissimum L.) varieties}

\author{
PC Yadav, RK Yadav, Vishawanath and Laxmi Prajapati
}

DOI: https://doi.org/ 10.22271/chemi.2020.v8.i4ai.10085

\begin{abstract}
A field experiment was conducted during Rabi season of 2015-16. To study the stability analysis of yield and its component traits in 20 genotypes of linseed i.e.; NPHY-29, NP-121, NPRR-271, NPRR-402, RL28-1, AHUDERA-170, NPHY-39, NPRR-28, NPHY-38, POLF-6, LCK-87312, RR-76, No-356, No-8, L-53, SJKO-05, No-3, 141No18 X RR-9, LMHS-397 and SJKO-55 will be grown in randomized block design with three replications at three different locations (Kanpur, Mainpuri and Mauranipur). The observations were recorded on some biological characters, viz.; 1000-seed weight (g), seed yield per plant (g), oil content (\%), protein content (\%), palmitic acid, stearic acid, oleic acid, linoleic acid and linolenic acid. In the present study of material SJKO-05 for 1000-seed weight (g), seed yield per plant (g) and protein content (\%) were found to be stable because $\mathrm{b}_{1}$ was near to unity, $\mathrm{S}^{2}$ di was not significant and mean values were more than population mean which was desirable. NP121 and NPRR 271 for palmitic acid, stearic acid, oleic acid and linolenic acid were found to be stable because b1 was near to unity, $\mathrm{S}^{2}$ di was not significant and mean values were less than population mean which was desirable.
\end{abstract}

Keywords: Linum usitatissimum, G X E interaction, Selection, Stability.

\section{Introduction}

Linseed (Linum usitatissimum L.) is a diploid ( $2 \mathrm{n}=30$, genome size $\sim 370 \mathrm{Mb}$ ) self-pollinated annual oilseed plant. It grown for both seed and fibre. Recently medical researchers have found linseed as best herbal source of carbohydrates, Omega- 3 and Omega- 6 fatty acids, which have beneficial effect on human metabolism. The seed of linseed is a very rich source of nutrients, which contain oil (40\%), protein (26\%), fibre (14\%), mucilage (12\%), water (9\%), minerals $(4 \%)$, potassium $(0.74 \%)$, phosphorous $(0.70 \%)$, magnesium $(0.38 \%)$, calcium $(0.21 \%)$, sulphur $(0.21 \%)$, along with sodium, chlorine, iron, zinc and copper in traces. Lignan (1.90-6.16 mg/g) is also found in the seed. The oil contains different fatty acids like linolenic (omega-3) 53.21\%, linoleic (omega-6) 17\%, oleic 18.51\%, stearic $4.42 \%$ and palmitic $4-6 \%$. Linseed is the richest source of omega- 3 fatty acid and it contains almost twice as much as of omega- 3 in fish oil. The ratio of omega- 3 and omega- 6 present in linseed is about $4: 1$, so this is a best herbal source of omega- 3 for improvement in human metabolism. World over, linseed is an important crop grown over 27.29 lakh ha with production of 25.20 lakh tons and average productivity of $923 \mathrm{~kg} / \mathrm{ha}$, while national production of 1.525 lakh tons is from 3.38 lakh ha area with low productivity of $473 \mathrm{~kg} / \mathrm{ha}$. As far as productivity is concerned, Indian average yield (473 kg/ha) is far below the productivity of UK (1500 kg/ha), Germany (1429 kg/ha), Canada (1538 kg/ha), USA (1076 kg/ha) and China (1000 kg/ha). India is an important linseed producer, which contributes about $11.82 \%$ to world acreage producing about $7 \%$ of world production. Anonymous (2015) ${ }^{[1]}$.

\section{Materials and Methods}

Basic material of present investigation comprising 20 pure lines of linseed i.e. NPHY-29, NP121, NPRR271, NPRR-402, RL-28-1, ADHUERA-170, NPHY-39, NPRR-28, NPHY-38, POLF-6, LCK-87312, RR-76, No-8, L-53, SJKO-05, No-3, 141No18XRR-9, LMHS-397 and SJKO-55 were grown in randomized block design with three replications at three different locations viz. Kanpur, Mainpuri and Mauranipur during Rabi 2015-16. Oil content was determined by Soxhlet method A.O.A.C 1975. There were analysed separately for their oil content and fatty acid composition. The data were reported about stability by Eberhart and 
Russell model of three replications. Methyl esters were prepared by the method of Luddy et al. (1968) ${ }^{[4]}$.

\section{Results and Discussion}

The stability analysis (Table 1 ) revealed highly significant differences among the genotype for all the characters except seed yield per plant and stearic acid. Seed yield per plant (g), protein content (\%) and stearic acid for environment. Similar result has been reported by Yadav et al. (2000-2014) ${ }^{[5,6,7]}$. 1000-seed weight (g), protein content (\%), palmitic acid, stearic acid, linoleic acid and linolenic acid for (E+GXE). Protein content (\%) and stearic acid for E(Linear). 1000-seed weight (g), seed yield per plant (g), oil content (\%), protein content (\%), palmitic acid, stearic acid, oleic acid and linolenic acid for GXE (Linear). 1000-Seed weight (g), seed yield per plant (g), oil content (\%), oleic acid, linoleic acid and linolenic acid for pooled deviation. Indicating substantial amount of genetic diversity in the material, such as observations were advocated by Eberhart and Russell model (1966) ${ }^{[3]}$ in their findings. Adaptability studies (Table-2) in linseed to considerable amount of G X E linear component emphasized genotypes deviating from regression line of unit slope could be identified. Accordingly, three kinds of linear component viz., $\mathrm{b}=1, \mathrm{~b}>1$ and $\mathrm{b}<1<0$ had been generally observed in all the characters. In the present study four genotypes namely, NPHY 29, NPRR 271, NPHY 38 and SJOK 05 for 1000-seed weight (g), Five genotypes namely, POLF 10, L-53, 141NO18XRR9, LMHS-5 and SJKO 55 for seed yield per plant (g), Six genotypes namely RL-28-1, NPRR 28, POLF 10, L-53, NO 3 and LMHS-5 for oil content (\%), Four genotypes namely, NP 121, NPHY 38, RR 76 and SJKO 55 for protein content (\%), Seven genotypes namely, NP121, NPRR 271, NPRR 402, NPHY 38, RR 76, 141NO18XRR9 and LMHS-5 for palmitic acid, Two genotypes namely, NPHY 29 and NPRR 271 for stearic acid, Twelve genotypes namely, NPHY 29. NPRR271, NPRR 402, NPHY 39, NPHY 38, POLF 10, NO 356, L-53, SJKO 05, NO141NO18XRR9 and SJKO 55 for oleic acid. All genotypes for linoleic acid showed significant regression coefficient $\left(b_{1}\right)$. Deviation from non-linear regression $\left(s^{2}{ }_{\mathrm{di}}\right)$ was significant in none of the genotypes and Nine genotypes namely, NPHY 29, NP 121, RL-28-1, AHUDERA 170, NPHY 39, POLF 10, NO 356, and LMHS-5 for linolenic acid were to be stable because $b_{1}$ was near to unity, $S^{2}$ di was not significant and mean values were less than population mean which was desirable. Such observation were reported by Alem and Dessalegn (2014) ${ }^{[9]}$; Temesgen et al. (2014) ${ }^{[10]}$; Vishnuvardhan and Rao (2014) ${ }^{[11]}$ and Yadav et al, (2014) ${ }^{[7]}$.

Table 1: Analysis of variance of stability for some biological characters in 20 parents in linseed (Linum usitatissimum L.).

\begin{tabular}{|c|c|c|c|c|c|c|c|c|c|c|}
\hline $\begin{array}{l}\text { Source of } \\
\text { variation }\end{array}$ & d.f. & $\begin{array}{l}\text { 1000- seed } \\
\text { weight }(\mathrm{g})\end{array}$ & $\begin{array}{c}\text { Seed } \\
\text { yield/plant }\end{array}$ & \begin{tabular}{|c|}
$\begin{array}{c}\text { Oil content } \\
(\%)\end{array}$ \\
\end{tabular} & $\begin{array}{c}\text { Protein } \\
\text { content }(\%)\end{array}$ & $\begin{array}{c}\text { Palmitic } \\
\text { acid }\end{array}$ & $\begin{array}{c}\begin{array}{c}\text { Stearic } \\
\text { acid }\end{array} \\
\end{array}$ & $\begin{array}{c}\text { Oleic } \\
\text { acid }\end{array}$ & $\begin{array}{c}\begin{array}{c}\text { Linoleic } \\
\text { acid }\end{array} \\
\end{array}$ & $\begin{array}{c}\begin{array}{c}\text { Linolenic } \\
\text { acid }\end{array} \\
\end{array}$ \\
\hline Genotype & 19 & $0.48^{* *}$ & 0.80 & $5.03^{* *}$ & $0.70^{*}$ & $41.26^{* *}$ & 1.96 & $157.53 * *$ & $121.56 * *$ & $242.05 * *$ \\
\hline Environment & 2 & $0.91 * *$ & 13.39 & $6.85^{* *}$ & 0.33 & $6.70 * *$ & 0.43 & 31.43** & $8.22 *$ & $17.82^{* *}$ \\
\hline$G \times E$ & 38 & 0.09 & 0.53 & 0.46 & 0.37 & 0.53 & 1.83 & 0.64 & 2.80 & 1.41 \\
\hline$E+G \times E$ & 40 & 0.14 & $1.17 * *$ & $0.78 *$ & 0.36 & 0.84 & 1.76 & $2.18 * *$ & 3.07 & 2.23 \\
\hline E (Linear) & 1 & $1.83 * *$ & $26.79 * * *$ & $13.71 * *$ & 0.66 & $13.41 * *$ & 0.86 & $62.87 * *$ & $16.45^{* *}$ & $35.64 * *$ \\
\hline G x E (Linear) & 19 & 0.12 & 0.66 & 0.58 & 0.48 & 0.16 & 1.47 & 0.48 & $3.87 *$ & 0.83 \\
\hline Pooled deviation & 20 & 0.07 & 0.38 & 0.32 & $0.24 *$ & $0.85 * * *$ & $2.08^{* * *}$ & 0.76 & 1.63 & 1.89 \\
\hline Pooled error & 114 & 0.10 & 0.33 & 0.59 & 0.12 & 0.09 & 0.009 & 0.57 & 1.35 & 1.26 \\
\hline
\end{tabular}

Table 2: Estimates of stability parameters based on three environments for some biological characters in linseed (Linum usitatissimum L.).

\begin{tabular}{|c|c|c|c|c|c|c|c|c|c|}
\hline \multirow{2}{*}{ Genotype } & \multicolumn{3}{|c|}{ 1000-Seed weight (g) } & \multicolumn{4}{|c|}{ Seed yield per plant (g) } & \multicolumn{2}{|c|}{ Oil content (\%) } \\
\hline & $\mathbf{x}^{-}$ & $\mathbf{b}_{\mathbf{i}}$ & $\mathbf{x}^{-}$ & $\mathbf{b}_{\mathbf{i}}$ & $S^{2} d_{i}$ & $\mathbf{S}^{2} \mathbf{d}_{\mathbf{i}}$ & $\mathbf{x}^{-}$ & $\mathbf{b}_{\mathbf{i}}$ & $\mathbf{S}^{2} \mathbf{d}_{\mathbf{i}}$ \\
\hline NPHY 29 & 7.05 & 0.95 & 36.29 & 0.66 & -0.45 & -0.104 & 5.83 & 0.28 & -0.27 \\
\hline NP 121 & 7.05 & 2.48 & 35.90 & 0.47 & 0.68 & -0.031 & 4.90 & 0.95 & -0.002 \\
\hline NPRR 271 & 6.94 & 1.07 & 36.62 & 1.71 & 0.10 & -0.102 & 5.29 & 1.01 & 0.49 \\
\hline NPRR 402 & 7.04 & $2.21 * *$ & 34.44 & 0.42 & 0.26 & -0.104 & 5.66 & 1.37 & -0.11 \\
\hline RL-28-1 & 6.38 & 0.69 & 37.21 & 1.00 & -0.55 & -0.102 & 4.61 & 0.50 & -0.18 \\
\hline AHUDERA 170 & 6.53 & 1.15 & 36.72 & $2.13^{* *}$ & -0.57 & -0.101 & 4.71 & -0.50 & 0.08 \\
\hline NPHY 39 & 6.39 & -0.005 & 36.55 & 1.30 & 0.33 & $-0.64 * *$ & 4.97 & 1.08 & -0.33 \\
\hline NPRR 28 & 7.04 & $2.21^{* *}$ & 37.04 & 1.53 & -0.50 & -0.104 & 5.21 & 1.04 & 0.62 \\
\hline NPHY 38 & 8.14 & 1.28 & 34.40 & $-0.52 *$ & -0.579 & -0.001 & 4.75 & 0.005 & 0.13 \\
\hline POLF 10 & 7.05 & 1.74 & 37.07 & 0.87 & -0.574 & 0.044 & 6.08 & 1.27 & -0.15 \\
\hline LCK 87312 & 6.71 & -0.38 & 36.73 & 1.19 & -0.44 & 0.016 & 5.71 & 0.87 & 0.52 \\
\hline RR 76 & 7.32 & 2.98 & 36.05 & 1.04 & -0.55 & 0.037 & 4.96 & 0.84 & $1.00 *$ \\
\hline NO 356 & 6.64 & 0.41 & 38.04 & 3.78 & 0.79 & -0.100 & 5.57 & 0.27 & -0.20 \\
\hline NO 8 & 6.94 & 2.71 & 36.73 & 0.43 & -0.34 & -0.054 & 5.86 & 2.18 & -0.28 \\
\hline L-53 & 6.62 & 0.63 & 40.00 & 0.70 & -0.54 & -0.096 & 6.05 & 1.41 & -0.027 \\
\hline SJKO 05 & 6.91 & 1.34 & 36.87 & -0.40 & -0.55 & -0.095 & 5.20 & 0.34 & 0.025 \\
\hline NO 3 & 6.59 & -0.20 & 39.06 & 1.41 & -0.33 & -0.082 & 6.23 & 2.26 & -0.19 \\
\hline 141NO18XRR9 & 6.36 & $-0.11^{*}$ & 36.32 & 0.60 & -0.57 & -0.104 & 5.93 & $1.60 *$ & -0.33 \\
\hline LMHS-5 & 6.76 & -1.32 & 37.99 & 1.29 & -0.11 & -0.099 & 5.30 & 1.55 & 0.27 \\
\hline SJKO 55 & 6.95 & 0.13 & 37.01 & 0.32 & -0.53 & -0.009 & 6.06 & 1.58 & 0.01 \\
\hline Population Mean & \multicolumn{3}{|c|}{6.87} & \multicolumn{3}{|c|}{5.44} & \multicolumn{3}{|c|}{36.85} \\
\hline SE Bi & \multicolumn{3}{|c|}{0.19} & \multicolumn{3}{|c|}{0.53} & \multicolumn{3}{|c|}{0.68} \\
\hline SE Mean & \multicolumn{3}{|c|}{0.89} & \multicolumn{3}{|c|}{0.43} & \multicolumn{3}{|c|}{0.40} \\
\hline
\end{tabular}


Table 3: contd.

\begin{tabular}{|c|c|c|c|c|c|c|c|c|c|}
\hline \multirow{2}{*}{ Genotype } & \multicolumn{3}{|c|}{ Protein content (\%) } & \multicolumn{4}{|c|}{ Palmitic acid } & \multicolumn{2}{|c|}{ Stearic acid } \\
\hline & $\mathbf{x}^{-}$ & $\mathbf{b}_{\mathbf{i}}$ & $\mathbf{x}^{-}$ & $\mathbf{b}_{\mathbf{i}}$ & $\mathbf{S}^{2} \mathbf{d}_{\mathrm{i}}$ & $S^{2} d_{i}$ & $\mathbf{x}^{-}$ & $\mathbf{b}_{\mathbf{i}}$ & $S^{2} d_{i}$ \\
\hline NPHY 29 & 15.68 & 5.07 & 3.93 & -1.17 & 0.022 & -0.11 & 6.39 & $0.74 *$ & -0.10 \\
\hline NP 121 & 16.15 & 0.57 & 3.11 & -0.88 & $0.223 * *$ & 0.0005 & 10.48 & 0.94 & 0.11 \\
\hline NPRR 271 & 15.58 & 4.93 & 4.15 & -1.32 & $0.075 * *$ & $0.45^{*}$ & 17.72 & $1.83 *$ & -0.09 \\
\hline NPRR 402 & 16.71 & -3.92 & 1.90 & 1.10 & $0.074 * *$ & -0.11 & 15.03 & 0.79 & 0.23 \\
\hline RL-28-1 & 15.37 & 1.35 & 4.18 & 4.41 & $17.62 * *$ & $0.48 *$ & 8.21 & 1.25 & $0.58^{*}$ \\
\hline AHUDERA 170 & 16.22 & 5.30 & 2.23 & 0.62 & $0.31^{* *}$ & -0.10 & 7.85 & 0.95 & 0.18 \\
\hline NPHY 39 & 16.27 & -0.12 & 3.36 & -4.32 & $0.26^{* *}$ & -0.05 & 6.16 & 1.00 & 0.03 \\
\hline NPRR 28 & 16.08 & $2.42 * *$ & 3.80 & -1.50 & $2.14^{* *}$ & -0.12 & 14.31 & 0.15 & $5.42 * *$ \\
\hline NPHY 38 & 16.98 & 1.87 & 4.27 & -2.06 & $1.56^{* *}$ & 0.34 & 9.04 & 1.48 & -0.03 \\
\hline POLF 10 & 16.01 & -0.87 & 4.02 & 8.98 & $3.09 * *$ & 0.35 & 7.34 & 1.39 & $0.85^{* *}$ \\
\hline LCK 87312 & 16.04 & -2.99 & 3.33 & -1.49 & $0.02 *$ & 0.002 & 3.54 & 0.56 & -0.01 \\
\hline RR 76 & 16.24 & 1.18 & 2.56 & 9.63 & $2.71 * *$ & 0.30 & 7.62 & 1.25 & $0.74 * *$ \\
\hline NO 356 & 15.41 & 10.79 & 4.07 & -11.24 & $0.29 * *$ & $0.46^{*}$ & 8.25 & 0.31 & $1.24 * *$ \\
\hline NO 8 & 16.22 & 2.26 & 4.40 & 7.38* & -0.008 & -0.09 & 6.39 & 0.48 & -0.07 \\
\hline L-53 & 14.79 & 1.44 & 4.63 & 2.31 & $2.38 * *$ & -0.02 & 4.72 & 1.04 & $1.14 * *$ \\
\hline SJKO 05 & 16.32 & $-3.60 *$ & 4.34 & 2.42 & $2.57 * *$ & -0.12 & 4.21 & 2.23 & $3.42 * *$ \\
\hline NO 3 & 15.78 & 1.33 & 4.50 & 6.34 & $0.10^{* *}$ & $0.42 *$ & 5.99 & 0.98 & 0.18 \\
\hline 141NO18XRR9 & 16.03 & -0.10 & 4.17 & 6.18 & $0.73^{* *}$ & -0.10 & 7.34 & 0.66 & $0.61 * *$ \\
\hline LMHS-5 & 16.04 & -6.15 & 3.46 & 6.19 & $1.04^{* *}$ & 0.53* & 11.65 & 0.77 & $0.59 * *$ \\
\hline SJKO 55 & 16.32 & -0.78 & 4.93 & -11.61 & $6.20 * *$ & -0.11 & 6.55 & 1.11 & 0.05 \\
\hline Population Mean & \multicolumn{3}{|c|}{16.01} & \multicolumn{4}{|c|}{8.44} & \multicolumn{2}{|c|}{3.77} \\
\hline SE Bi & \multicolumn{3}{|c|}{2.70} & \multicolumn{4}{|c|}{1.13} & \multicolumn{2}{|c|}{6.94} \\
\hline SE Mean & \multicolumn{3}{|c|}{0.35} & \multicolumn{4}{|c|}{0.65} & \multicolumn{2}{|c|}{1.02} \\
\hline
\end{tabular}

Table 4: contd.

\begin{tabular}{|c|c|c|c|c|c|c|c|c|c|}
\hline \multirow{2}{*}{ Genotype } & \multicolumn{3}{|c|}{ Oleic acid } & \multicolumn{2}{|c|}{ Linoleic acid } & \multicolumn{4}{|c|}{ Linolenic acid } \\
\hline & $\mathbf{x}^{-}$ & $\overline{b_{i}}$ & $\mathbf{x}^{-}$ & $\mathbf{b}_{\mathbf{i}}$ & $\mathbf{S}^{2} \mathbf{d}_{\mathrm{i}}$ & $S^{2} d_{i}$ & $\mathbf{x}^{-}$ & $\mathbf{b}_{\mathbf{i}}$ & $\mathbf{S}^{2} \mathbf{d}_{\mathrm{i}}$ \\
\hline NPHY 29 & 23.16 & 0.66 & 58.79 & 0.78 & -1.22 & -0.54 & 7.71 & 0.69 & -0.91 \\
\hline NP 121 & 9.40 & 0.89 & 65.25 & 1.05 & -1.12 & -0.50 & 11.73 & 1.02 & -1.17 \\
\hline NPRR 271 & 21.04 & $1.29 *$ & 41.72 & 1.48 & -0.35 & -0.56 & 15.34 & 2.36 & -1.22 \\
\hline NPRR 402 & 26.38 & 0.90 & 36.63 & 1.23 & -0.95 & -0.44 & 20.85 & -0.68 & 3.74 \\
\hline RL-28-1 & 18.37 & 1.11 & 57.26 & 1.08 & -1.22 & -0.53 & 12.62 & 3.03 & 1.73 \\
\hline AHUDERA 170 & 12.55 & 2.30 & 64.93 & 1.44 & -0.84 & $2.80^{*}$ & 12.41 & 1.68 & 2.22 \\
\hline NPHY 39 & 23.03 & $0.72 *$ & 57.78 & 1.20 & -0.88 & -0.56 & 9.64 & 1.91 & -0.52 \\
\hline NPRR 28 & 5.07 & 0.92 & 39.71 & 0.98 & -1.22 & -0.54 & 36.86 & -0.35 & -1.21 \\
\hline NPHY 38 & 25.32 & 0.71 & 43.84 & 1.03 & -1.10 & -0.55 & 17.51 & 2.69 & -0.37 \\
\hline POLF 10 & 21.29 & 1.01 & 55.81 & 0.95 & -1.22 & -0.54 & 12.44 & -2.40 & $8.60 * *$ \\
\hline LCK 87312 & 10.81 & 1.18 & 71.17 & 1.78 & $32.32 * *$ & $9.86 * * *$ & 11.14 & -0.70 & $4.25^{*}$ \\
\hline RR 76 & 19.34 & 1.03 & 59.04 & 0.96 & -1.21 & -0.39 & 11.39 & -1.13 & -1.11 \\
\hline NO 356 & 20.81 & 1.01 & 57.82 & 1.06 & -1.22 & 0.11 & 8.67 & $2.83 *$ & -1.33 \\
\hline NO 8 & 23.19 & 0.15 & 48.90 & 2.15 & -0.84 & -0.55 & 17.12 & -3.87 & 0.96 \\
\hline L-53 & 24.49 & 1.26 & 54.09 & -0.46 & -0.80 & -0.54 & 11.92 & $2.69 * *$ & -1.34 \\
\hline SJKO 05 & 30.27 & 0.92 & 47.91 & 1.15 & -1.02 & -0.55 & 13.51 & 2.74 & -1.16 \\
\hline NO 3 & 32.57 & 1.00 & 48.52 & 1.22 & -1.258 & -0.51 & 8.26 & -0.17 & -1.33 \\
\hline 141NO18XRR9 & 29.42 & 1.05 & 49.30 & 1.02 & -1.251 & -0.45 & 9.14 & 0.26 & -134 \\
\hline LMHS-5 & 13.34 & 0.88 & 58.63 & -1.02 & -0.64 & -0.48 & 12.84 & 1.96 & -1.29 \\
\hline SJKO 55 & 23.09 & 0.90 & 50.56 & 0.85 & -1.25 & -0.49 & 14.85 & $5.43 *$ & -1.33 \\
\hline Population Mean & \multicolumn{3}{|c|}{20.65} & \multicolumn{2}{|c|}{13.80} & \multicolumn{4}{|c|}{53.38} \\
\hline $\mathrm{SE} \mathrm{Bi}$ & \multicolumn{3}{|c|}{0.49} & \multicolumn{2}{|c|}{1.41} & \multicolumn{4}{|c|}{0.97} \\
\hline SE Mean & \multicolumn{3}{|c|}{0.61} & \multicolumn{2}{|c|}{0.90} & \multicolumn{4}{|c|}{1.03} \\
\hline
\end{tabular}

\section{References}

1. Anonymous. Food and Agriculture organization of the United Nation. FAO Statistics, 2014-15. (www.faostat.org.com).

2. Anonymous. methods of analysis, $12^{\text {th }}$ edition, Association of official analytical chemists, Washington, D.C, 1975.

3. Eberhart SA, Russell WA. Stability parameters for comparing varieties. Crop Sci. 1966; 6:36-40.

4. Luddy EF, Barforad RA, Berbs SF, Magidman P. J Amer Oil Chem Soc. 1968; 65:549.
5. Yadav RK, Singh PK, Gupta RR. Phenotypic stability for yield and quality attributes in linseed (Linum usitatissimum L.). Crop Res. 2000; 19(2):301-304.

6. Yadav RK. Association studies over locations in linseed (linum usitatissimum L.) Prog. Agic. 2001; 1(1):11-15.

7. Yadav RK, Yadav AK, Shweta Singh L, Verma PN. Stability analysis in linseed (Linum usitatissimum) varieties. Indian Journal of Agricultural Science. 2014; 84(7):883-886.

8. Jinks JL, Perkins JM, Breeze EI. A general method of detecting dominance and epistatic variation for metric 
traits II. Application to inbred lines. Heredity. 1969; 24:45-57.

9. Alem C, Dessalegn T. Study on genotype x environment interaction of seed yield, oil content, fatty acid profile and stability analysis of yield related trait in linseed (Linum usitatissimumL.). International Journal of Plant Breeding and Genetics. 2014; 8(2):66-73.

10. Temesgen A, Mammo K, Lule D. Genotype by environment interaction $\left(\begin{array}{lll}G & x & E\end{array}\right)$ and grain yield stability analysis of Ethiopian linseed and Niger seed varieties. Journal of Applied Bioscience. 2014; 80:7093-7101.

11. Vishnuvardhan KM, Rao SS. Stability analysis for yield and its attributing characters in linseed (Linum usitatissimum L.). International Journal of Plant, Animal and Environmental Sciences. 2014; 4(2):408-412.

12. Tewari N, Singh N Shweta. Selection parameters for seed yield and its components in linseed (Linum usitatissimum L.). Current Advances in Agricultural Sciences. 2012; 4 (2):149-151. 